\title{
Transoral Robotic Surgery for Residual and Recurrent Oropharyngeal Cancers
}

\author{
Vinidh Paleri, MS, FRCS ${ }^{a, b, *}$, John Hardman, MSc, MRCS ${ }^{a, c}$, \\ Grainne Brady, MRes, MRCSLT ${ }^{\mathrm{d}}$, Ajith George, FRCS ${ }^{\mathrm{e}, \mathrm{f}}$, \\ Cyrus Kerawala, FDSRCS, FRCS ${ }^{a}$
}

\section{KEYWORDS}

- Recurrence • Cancer • Robotics • Surgery • Head and neck

\section{KEY POINTS}

- Transoral robotic surgery (TORS) for residual, recurrent tumors and new primaries in radiation-exposed fields is becoming increasingly adopted as more centers gain access to robotic systems and as favorable outcome data emerge.

- Achieving clear resection margins can be technically challenging in these cases and a TORS program should be considered only by experienced and appropriately trained surgeons.

- Transoral reconstruction additionally may be required and presents its own technical complexities.

- Successful functional outcomes are achievable but require a well-resourced and motivated team to manage patients' expectations and to support them through a potentially prolonged period of rehabilitation.

\section{INTRODUCTION}

The most widely used treatment of the management of residual, recurrent, and new primary radiation-exposed (ReRuNeR) oropharyngeal cancer (OPC) is open surgery, with or without reconstruction. ${ }^{1-4}$ Open surgery is prolonged, involves significant disruption to normal anatomy to gain access to the tumor (mandibulotomy, floor of mouth dissection, and lingual release), almost always needs reconstruction, and increases recovery time. Additionally, the irradiated bone can be beset with healing

\footnotetext{
a Head and Neck Unit, The Royal Marsden NHS Foundation Trust, Fulham Road, London SW3 6JJ, UK; ${ }^{b}$ The Institute of Cancer Research, Brompton Road, London SW3 6JJ, UK; ${ }^{c}$ North London, UK; ${ }^{d}$ Department of Speech, Language and Swallowing, The Royal Marsden NHS Foundation Trust, Fulham Road, London SW3 6JJ, UK; ${ }^{~}$ University Hospitals North Midlands, North Staffordshire, England; ${ }^{\dagger}$ Keele University Medical School, Staffordshire, UK

* Corresponding author.

E-mail address: vinidh.paleri@rmh.nhs.uk

Twitter: @VinPaleri (V.P.)
} 
issues in nearly half of patients. ${ }^{5}$ A transoral robotic surgery (TORS) approach abrogates these disadvantages. The superior ability to maneuver instruments in a confined space and perform an en bloc resection makes TORS-based approaches a viable option for ReRuNeR OPCs.

Salvage surgery continues to be the most effective treatment modality in ReRuNeR OPCs, as demonstrated in the systematic review by Jayaram and colleagues. ${ }^{2}$ Recent studies show that the difference in survival can be as much as $50 \%,{ }^{6}$ with salvage surgery reducing the risk of death from residual cancers by half. ${ }^{7}$ In the human papillomavirus (HPV)-positive squamous cell carcinoma population, recurrence at the primary site is uncommon and seen in only $5 \%$ to $7 \%$ of patients after intensity-modulated radiation therapy, ${ }^{8,9}$ because most index tumors are of early stage with excellent Q12 $_{12}$ response rates to primary surgery or nonsurgical treatments. ${ }^{10} \mathrm{HPV}$-negative squamous cancers are more likely to recur at the primary site, but higher rates of comorbidities in this group may reduce their tolerance of any postoperative aspiration. As such, a smaller proportion of the HPV-negative, compared with HPV-positive, patients may be suitable for salvage surgery. All things considered, patients with ReRuNeR cancers may not be offered salvage surgery, given the significant morbidity of open surgery, resource utilization, and the wide perception of relatively poor outcome.

Significant expertise in TORS has been accrued since its use has been described in the management of treatment-naïve cancers in early years of this century. The experience with TORS for recurrent cancer has been described in single centers and also has been the subject of a systematic review and meta-analysis by the authors' group. ${ }^{11}$ The oncological and functional outcomes of TORS for recurrent cancer are $\mathrm{Q}_{13}$ supportive and, in carefully selected patients, transoral resection is an acceptable procedure to perform with satisfactory functional outcomes.

This article aims to offer readers the principles of case selection, decision making, tips on robotic surgical resection of these cancers, principles of reconstruction, rehabilitation, and future trends for using TORS in the management of ReRuNeR OPCs. Q14 Although the clinical and technical aspects and functional outcomes are based on the authors' experience, the oncological outcome data discussed are based on the systematic review and meta-analysis published recently by the authors' group. ${ }^{11}$

\section{ASSESSMENT OF RESIDUAL, RECURRENT, AND NEW PRIMARY RADIATION- EXPOSED OROPHARYNGEAL CANCERS FOR TRANSORAL ROBOTIC SURGERY}

The authors recommend an assessment of resectability be performed under general anesthesia for all patients with ReRuNeR OPCs deemed suitable for surgery. During assessment, the surgeon should ensure that the full mucosal extent of the tumor is visible using the robotic telescope and appropriate retractors, including a tongue stitch for traction. It is the authors' experience that a general anesthetic assessment often converts a tumor previously considered unresectable, based on clinical assessment, to amenable to resection via a TORS approach.

Special consideration should be given to the impact of trismus on access, especially if the lower extent of the tumor is not visible after robotic docking. Compounded by individual anatomy (long neck, narrow mandibular arch, and retrognathia), even mild trismus can make dissection of the anteroinferior aspects of the tumor difficult, with poor visualization and frequent instrument clashes resulting from the 3 instrument arms competing for space. If free flap reconstruction is planned (discussed later), adequate access is essential to allow for flap inset. Currently, the only available needle drivers are relatively large, at $8 \mathrm{~mm}$, making suturing of the lower aspect of the flap challenging when space is limited. In the authors' experience, the most common 
reason for considering excision via conventional open surgery, rather than TORS, is trismus. The authors' baseline preoperative functional parameters at regarding trismus and swallow are discussed.

\section{T STAGE AND TRANSORAL ROBOTIC SURGERY}

TORS is approved as a treatment modality for early-stage oropharyngeal tumors in the primary setting and is bolstered by a considerable evidence base. Given the advantages of TORS over open surgery, however, the authors believe that, in select cases, TORS also may be suitable for tumors with higher T classifications. This is supported ${ }_{015}$ by data from the authors' meta-analysis, ${ }^{11}$ in which $8.3 \%$ of the recurrent tumors identified were staged T3 or T4. In the authors' initial series (Paleri V, Fox H, Coward S, et al. Transoral robotic surgery for residual and recurrent oropharyngeal cancers: an IDEAL phase 2a exploratory study of surgical innovation. In: Unpublished, ed., 2016), $23 \%$ of en bloc resections measured $6 \mathrm{~cm}$ or more, indicating that larger tumors can be removed via a TORS approach.

\section{THE TREATING TEAM AND EXPERIENCE WITH PRIMARY TRANSORAL ROBOTIC SURGERY}

TORS is associated with a well-recognized learning curve. The authors caution against the use of TORS in ReRuNeR OPCs for surgeons who are early in their learning curve, especially if they have had limited experience with nonrobotic transoral surgery previously. Significant clinical judgment is needed to select appropriate cases, and the experience accrued from primary TORS is crucial to delivering a good outcome in this patient group. The postoperative course for these cancers differs from that of primary resections, and the experience the wider team gleans during the learning curve, notably in postoperative management and rehabilitation, is invaluable in counseling and caring for patients with ReRuNeR OPCs. As a guide, the authors recommend undertaking 30 primary cases, with careful assessment of postoperative outcomes and margin status, before embarking on a ReRuNeR cancer program. ${ }^{12}$

\section{RELEVANT ANATOMY FOR MULTISITE OROPHARYNX RESECTIONS}

Most recurrences, especially those of the tongue base, do not necessarily fall within the compartmental resection of lateralized tongue base tumor, as described by Weinstein and O'Malley. ${ }^{13}$ If a tumor in the second subsite is superficial, then extension of the resection to the second subsite to take the tumor, a margin of mucosa, and a few millimeters of deeper tissue, should be relatively straightforward. If a tumor has substantial depth across both the tonsil and tongue base or extends deeply into the tonsillolingual sulcus, however, a greater appreciation of the deeper anatomy is warranted. In order to achieve an en bloc resection of these tumors, or tumors extending into the laryngopharynx, the surgeon must be able to identify the following structures from a transoral approach: the styloglossus muscle, the hyoid and its constrictor muscle attachments, and branches of the facial ${ }^{14}$ and lingual arteries. ${ }^{15}$

Broadly, the pharyngeal constrictors are separated into 3 groups of muscles. The inferior constrictor is recognized as being divided into 2 elements, which are not relevant in transoral surgery. The subdivisions of the superior and middle constrictors are ${ }_{016}$ particularly important, however, when dissecting the parapharyngeal space. The superior constrictor is made up of 5 muscle slips: pterygopharyngeus, palatopharyngeus, buccopharyngeus, myelopharyngeus, and glossopharyngeus (Fig. 1). The window to the inverted tetrahedron of the parapharyngeal space is the 


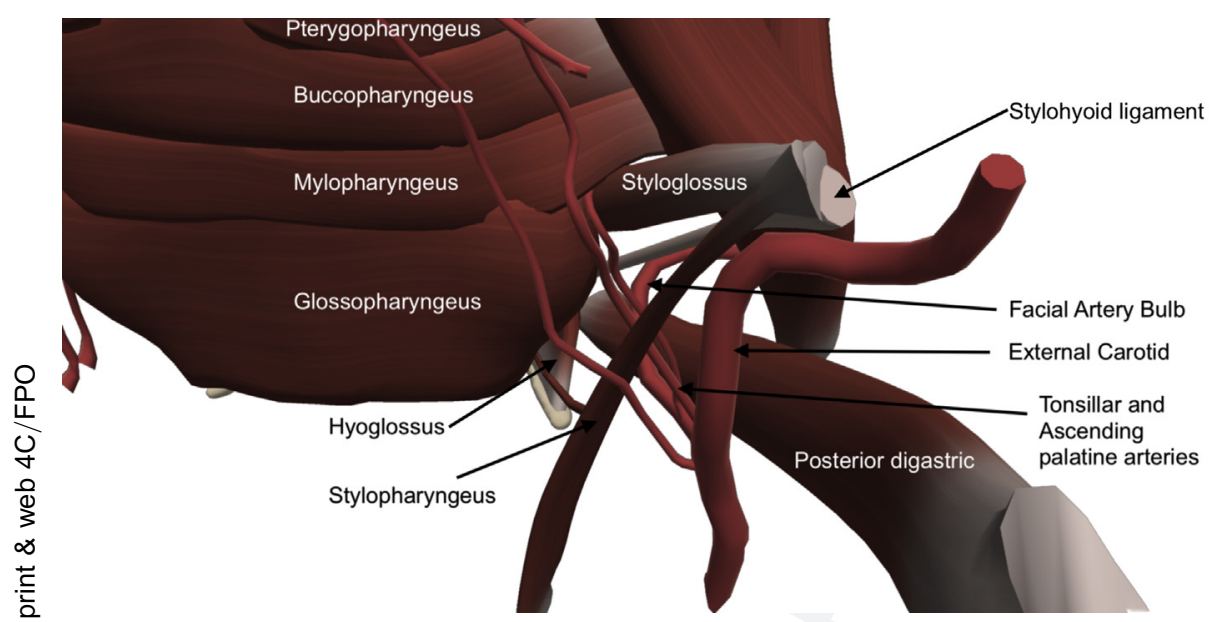

Fig. 1. Schematic from a right posterolateral view showing the various superior constrictor slips, the styloglossus muscle, and the vascular anatomy relevant to TORS in the lateral pharynx.

pterygomandibular raphe. The raphe is the junction of the buccinator and the buccopharyngeus slip of the superior constrictor.

The styloglossus anatomy, as relevant to TORS, has been well described. ${ }^{16}$ The styloglossus muscle merges with the glossopharyngeus, the most inferior slip of the superior constrictor. The facial and lingual branches of the external carotid are consistently lateral to the plane of the styloglossus. The 2 main branches of the facial artery, which supply the tonsils (ascending palatine and tonsillar branches) can pass either above or below the styloglossus. When passing below the styloglossus, they can either be in the space between the styloglossus and stylopharyngeus (most common variation) (see Fig. 1) or deep to both muscles. Dissecting lateral to styloglossus, the facial artery bulb ${ }^{14}$ is the first major vessel to be encountered in the parapharyngeal space and often is mistaken for the lingual artery (Fig. 2). The bulb takes form as it arches over the posterior belly of digastric before heading into the substance of the submandibular gland (see Fig. 1). Encountering this vessel inevitably means a communication is created between the transoral and transcervical dissections, if performed concurrently.

If dissection continues caudally, in a plane lateral to styloglossus, then the superior thyroid artery is encountered. More medially, an early branch of the superior thyroid artery, the superior laryngeal artery, is seen as it courses through the pharyngoepiglottic fold; this vessel can be readily controlled with a clip applicator, early in the dissection of the fold, if reduced blood flow to the larynx is desired for the perioperative course.

Inferior to the glossopharyngeus, and where the styloglossus has merged with it anteriorly, the surgeon is guided by the 2 muscle slips of the middle constrictor. These are the ceratopharyngeus (attaches to the greater cornu) and chondropharyngeus (attaches to the lesser cornu) (Fig. 3). Their attachments to the hyoid are relevant as they pass medial to the hyoglossus. The lingual artery passes from lateral to medial proximal to where the chondropharyngeus attaches to the posterior aspect of the lesser cornu of the hyoid. After giving off the dorsal lingual branch, the artery courses toward the deep tongue musculature, entering in the plane between genioglossus and the 
202

203

204

205

206

207

208

209

210

211

212

213

214

215

216

217

218

219

220

221

222

223

224

225

226

227

228

229

230

231

232

233

234

235

236

237

238

239

240

241

242

243

244

245

246

247

248

249

250

251

252

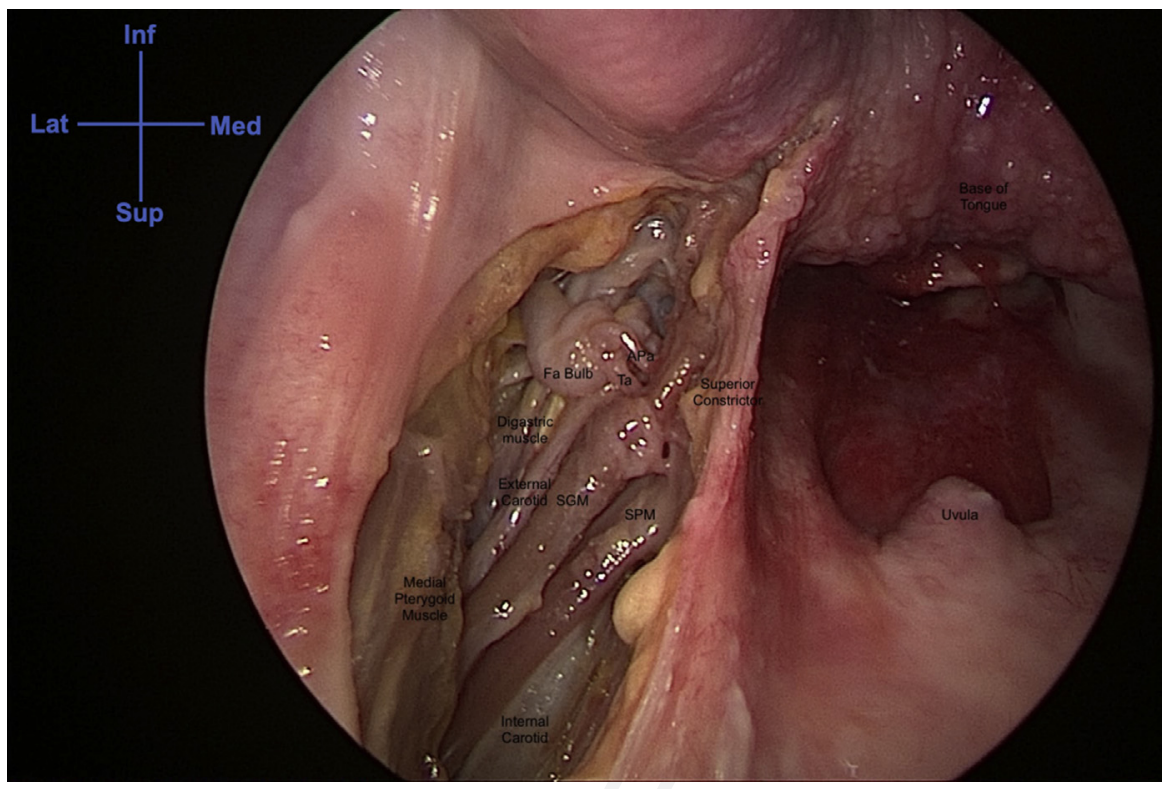

Fig. 2. A cadaver dissection of the left parapharyngeal space demonstrating the anatomy lateral to the styloglossus muscle.

lingual tonsils, where it is encountered during a conventional tongue base resection. Lateral to the hyoglossus muscle, almost mirroring the lingual artery from the transoral perspective, lies the hypoglossal nerve (Fig. 4).

\section{SURGICAL TECHNIQUE AND REFINEMENTS Neck and Airway}

In all cases, the ipsilateral neck should be explored to ligate the facial, lingual and ascending pharyngeal arteries; if there is concurrent metastatic neck disease, a

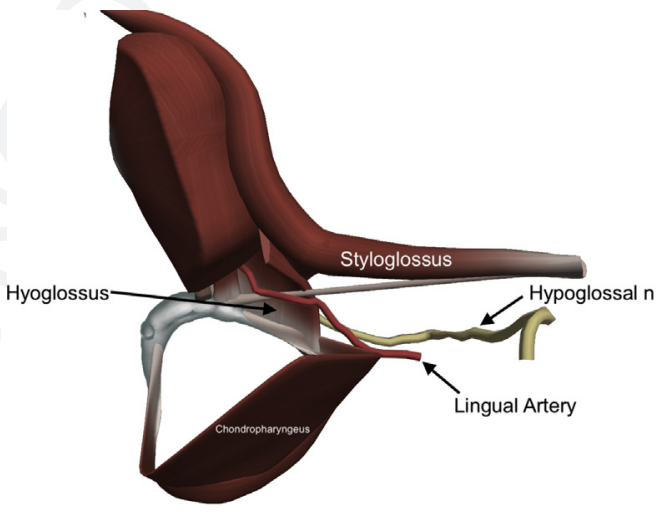

Fig. 3. Schematic showing the chondropharyngeus slip and its relation to the hyoglossus muscle and the lingual artery. 
253

254

255

256

257

258

259

260

261

262

263

264

265

266

267

268

269

270

271

272

273

274

275

276

277

278

279

280

281

282

283

284

285

286

287

288

289

290

291

292

293

294

295

296

297

298

299

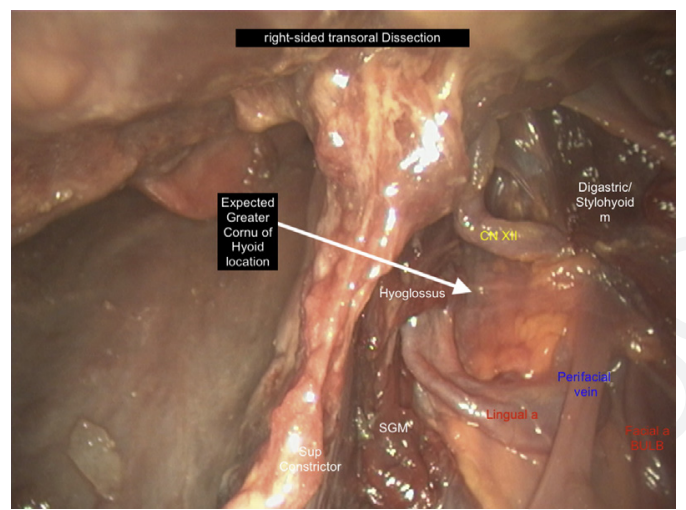

Fig. 4. A cadaver dissection of the right parapharyngeal space demonstrating the anatomy lateral and caudal to the styloglossus muscle, where the hyoglossus and the XII cranial nerve (CN) can be seen.

neck dissection may need to be performed. Given the low incidence of occult metastasis, the authors do not routinely perform an elective neck dissection for recurrent cancers, except in cases of free flap reconstruction, where vascular access is required for microvascular anastomosis and limited lymph nodal clearance is needed to accommodate the recipient vessels. The authors' preference is to perform a tracheostomy for tongue base ReRuNeR tumors because this improves transoral access and is retained for the immediate postoperative period as a safety to cover the known bleeding risk in this population (discussed later). Smaller ReRuNeR OPCs, such as tumors confined to the tonsil, may be resected with an oral endotracheal tube in situ, but this should be sutured to the contralateral oropharynx to prevent interaction with the operative field and instruments.

\section{Retractor Choice}

The Boyle-Davis retractor and the FK-WO retractor commonly are used for oropharyngeal resections. If both are available, the choice of retractor is influenced principally by the access required and the size of the tumor. The authors find the Boyle-Davis retractor useful for small tonsil tumors, without extension to the tongue base. In such cases, the convex profile of the Doherty blade is less problematic for instruments passing the oral cavity, and the smaller retractor gives excellent access for the first assistant at the bedside. The authors find the FK-WO retractor appropriate for a majority of other ReRuNeR OPCs undergoing TORS; its various blade attachments and adjustability allow finessed manipulation of the tongue to optimize instrument access and tumor retraction.

\section{Tonsil Recurrence}

In patients with ReRuNeR OPCs confined to the tonsillar fossa, the technique for resection is similar to the radical tonsillectomy described by Weinstein and colleagues. ${ }^{17}$ In more advanced cases, where the tonsillar tumor extends into the tongue base, the resection can include the affected tongue musculature and floor of mouth mucosa, using the anatomic principles discussed previously.

\section{Tongue Base Recurrence}

When TORS for ReRuNeR tongue base tumors is compared with its use in the primary setting, 2 major differences emerge: (1) recurrent tumors often extend anterior to the 
circumvallate papillae in a submucosal plane, rendering the anterior part of the tumor is readily palpable, and (2) the tongue base tissues often are edematous and brawny as a result of the previous irradiation. These tissue changes are problematic particularly for the operating surgeon: first, it is hard to discriminate tumor from the surrounding normal tissue at the mucosal level because the visual usual haptic feedback that is helpful in primary TORS is masked; and, second, the interaction of the monopolar cautery with the noncancerous deeper tissue appears more similar to that of cancerous tissues. Both these differences are primarily responsible for the proposed technical refinements, outlined in Paleri and colleagues (Paleri V, Fox H, Coward S, et al. Transoral robotic surgery for residual and recurrent oropharyngeal cancers: an IDEAL phase 2a exploratory study of surgical innovation. In: Unpublished, ed., 2016).

The current robotic retractors and set up are neither required nor adaptable for surgery anterior to the circumvallate papillae, where direct transoral access can be obtained easily, negating the need for robotic instrumentation in this area. In such cases, a stitch is placed on the anterior aspect of the tongue, as for other TORS resections, and the tongue is pulled forward. The anterior margin of the palpable tumor is marked out with methylene blue. Using monopolar cautery and digital palpation, an anterior shelf is established to separate the base of the tongue affected by tumor from the unaffected anterior tongue (Fig. 5A). This shelf is extended as needed to establish and define the tumor depth, allowing, as far posteriorly as possible, for an adequate margin of normal tissue in all dimensions. With progressive anterior and medial mobilization, a surprisingly significant amount of dissection can be performed transorally with a headlight and monopolar cautery. For dissections further posteriorly, a 6-in insulated monopolar blade can be used (Conmed, Utica, New York). Laterally, a19 these ReRuNeR tumors often extend superficially to the free margin of the tongue, and the deep margin may be formed by the posterior floor of mouth/sublingual glands. Removal of these structures may therefore be required for adequate clearance.

Once a substantial shelf is established and medial dissection as far back as possible has been performed, the FK-WO TORS retractor, with the mandible or WO blade, is placed in such a way that the blade of the retractor is anchored into the surgically created shelf; the tumor is now in the oropharyngeal lumen and the surgeon also
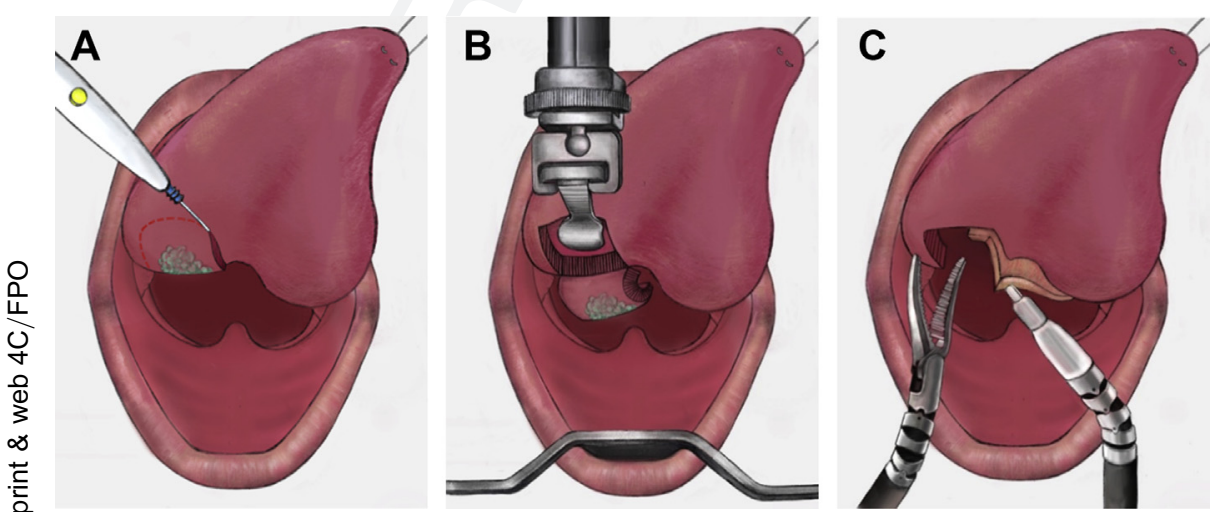

Fig. 5. (A) Creation of an anterior shelf that separates the base of the tongue from the anterior aspect of the tongue, using monopolar cautery and digital palpation $(B)$ The FK-WO TORS retractor with the mandible blade anchored into the surgically created shelf, allowing the tumor to drop behind the blade into the oropharyngeal lumen. (C) Resection where the tongue base, vallecula, and ipsilateral epiglottis have been resected. 
has defined the tumor depth (Fig. 5B, C). This maneuver gains additional space in the oral cavity for the robotic arms, because the mandible blade rests in the space occupied by the tongue base tumor and allows the tumor to progressively drop down into the lumen. The tumor usually is mobilized sufficiently at this point to allow the robotic arms to manipulate the tumor, aiding further dissection. In the early part of the learning curve, after incremental resection, the authors recommend that the robot be undocked repeatedly to allow the surgeon to confirm the tumor depth by direct palpation. Incremental resection, following this technique, allows progressive mobilization of the tumor, resulting in more accurate assessment of the adequacy of the resection margins.

\section{Tonsillolingual Sulcus Recurrence}

In the authors' early experience, they found deep-seated tumors in this area to be the most difficult for performing en bloc resections, given the need to resect both the tonsil and tongue base to a sufficient depth. In these instances, an appropriate margin of the tonsil is marked out, and the parapharyngeal space is entered. The surgeon must be able to define the parapharyngeal space, even when approaching it through the tonsil, because conventional access through the pterygomandibular raphe leads to an unnecessarily large resection. Once the styloglossus is identified, dissection can proceed laterally and inferiorly to it, as required, until the middle constrictor and the hyoid are identified. Appropriate tongue base cuts, as described in section 5.4, are performed around the tumor anteriorly, and the dissection proceeds to the required depth using the styloglossus dissection laterally as a guide. These techniques allow for an en bloc resection of these tumors (Fig. 6).

\section{Intraoperative Ultrasound}

Intraoperative ultrasound is useful in select instances, especially with submucosal tongue base cancers where intraoperative examination does not allow for a good assessment of the tumor extent and depth (Paleri V, Fox H, Coward S, et al. Transoral robotic surgery for residual and recurrent oropharyngeal cancers: an IDEAL phase $2 \mathrm{a}$ exploratory study of surgical innovation. In: Unpublished, ed., 2016). ${ }^{18}$ The Flex Focus 800 machine (BK Medical, Peabody, Massachusetts), with the robotic drop-in ultrasound transducer 8826 , is the authors' preferred instrument for tongue base lesions. After appropriate retractors are used to expose the tumor, the ultrasound transducer is
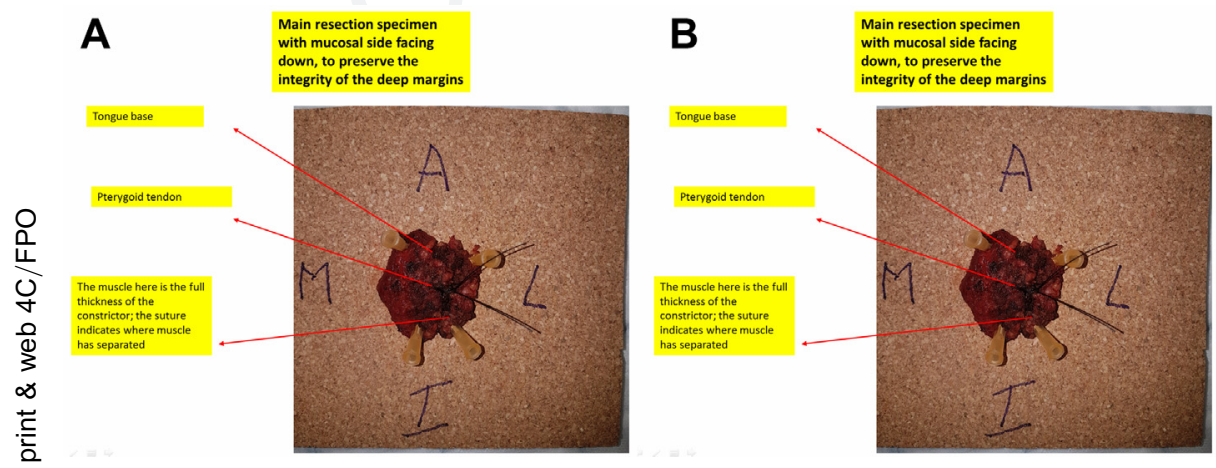

Fig. 6. The resected specimen is orientated and mounted with the deeper side facing up, taking care not to distort the convexity. (A) Shows the annotated mucosal side of the tumor before being orientated using pins $(B)$. 
grasped by the Maryland forceps and placed on the mucosal surface of the tongue base. In combination with the patient's preoperative magnetic resonance imaging, the transoral ultrasound images of the tongue base are interpreted by the radiologist and TORS surgeon in combination as the tumor resection progresses.

\section{Intraoperative Margin Assessment}

It is reasonable to aim to achieve a 5-mm margin for the deep and mucosal resections where the anatomy allows. It is the authors' opinion that a greater emphasis should be placed on achieving a clear margin clinically, rather than focusing on the numerical figure attached to the resection specimen in the final pathology report. This is relevant particularly because the final reading may reduce by up to $25 \%$, resulting from tissue necrosis induced by the energy devices commonly used in TORS, and up to $10 \%$ further when undergoing formalin fixation. ${ }^{19,20}$

For tonsil cancers, a 5-mm mucosal margin is achievable in most cases; but, these margins may not be possible for the deeper aspect of the mobilized specimen laterally, where the thickness of the constrictor bed measures less than $2 \mathrm{~mm}$, reduced by radiation induced atrophy and formalin fixation. Smaller tongue base cancers may achieve 5-mm deep and mucosal margins if they are confined to this subsite. For tumors centered in the tonsillolingual sulcus, the deep margin may be augmented by including the constrictor and stylohyoid muscles in the resected specimen.

It is important for the surgeon to examine all aspects of the specimen once it is completely resected. The authors do not routinely slice the specimen in the operative room to assess the deep margin. Areas where the deep resection margin is felt to be close are marked with colored ink on the specimen. The specimen then is orientated and temporarily placed back into the defect, pinpointing the precise location for further resection. Frozen section examination then can be used to confirm adequate clearance. For small oropharyngeal defects, the whole tumor bed may be sampled as a single marginal biopsy if required.

\section{Intraoperative Frozen Section}

Significant input from pathology services is needed to run such a service but it can be invaluable in the management of ReRuNeR OPCs: in some cases, a definitive confirmation of malignant disease may be required before progressing with a more advanced resection, especially where chronic ulceration has affected the oropharyngeal tissues and previous attempts at more superficial biopsy have been ambiguous; in other cases, the full submucosal extent of the tumor is difficult to appreciate in the irradiated tissue and histologic confirmation of adequate resection is needed to avoid removing unaffected tissue.

\section{Specimen Processing}

Special attention should be paid to the mounting of specimens to allow for accurate margin assessment. Traditionally, specimens are orientated, pinned, and mounted with the mucosal side facing up. If this method is applied to the en bloc oropharyngeal specimen resected with TORS, then the convex shape of the deep aspect may be lost when undergoing formalin fixation while flattened against the board. Additionally, once fixed in formalin, the muscle layers on the under-surface of the tumor specimen, that were freely mobile in vivo, have become compressed and distorted, which may contribute to an underestimation of these margins. Consequently, the authors recommend that the specimen is mounted with the mucosal side facing down, to maintain the natural convexity of the deeper aspect of the resected specimen (see Fig. 6). Furthermore, to ensure optimal communication between surgeons and 
histopathologists, it is the authors' practice to photograph all resections and provide labeled diagrams to facilitate specimen orientation prior to processing.

\section{Reconstructive Strategy}

461

462

463

464

465

466

467

468

469

470

471

472

473

474

475

476

477

478

479

480

481

482

483

484

485

486

487

488

489

490

491

492

493

494

495

496

497

498

499

500

501

502

503

504

505

506

507

TORS oropharyngeal resections traditionally have been left to heal by secondary intention but in the salvage setting complex ablative defects often cross a variety of anatomic subsites and, as a result, may require formal reconstruction. The anatomic goals of such reconstruction include coverage of vital vascular structures and maintenance of a watertight seal. Additionally, there are functional goals, such as minimizing velopharyngeal insufficiency and improving swallow by restoring tongue volume. These aims can be achieved by the transfer of vascularized tissue either locally or more often as a microvascular free flap. ${ }^{21-23}$

Reconstructive algorithms have been developed to aid planning of post-TORS resection defects. de Almeida and colleagues ${ }^{22}$ suggested 4 classes of defect: class I involves 1 subsite (tonsil, tongue base, pharynx, or soft palate) and no adverse features (internal carotid artery exposure, neck communication, or $>50 \%$ of soft palate resection); class II is similar but involves more than 1 subsite; class III involves 1 subsite but has 1 or more adverse features; and class IV involves multiple subsites and adverse features. Class I and class II defects can be either left to heal by secondary intention or closed with local flaps, whereas class III and class IV defects require regional or free flap reconstruction.

Oropharyngeal surgery in the salvage setting has shifted toward minimally invasive approaches, such as TORS, which aim to avoid unnecessary tissue disruption from access procedures. The natural evolution of this progression has been the development of robotic-assisted free flap inset (RAFFI). Combining resection and reconstruction robotically is a more cost-effective and efficient use of the robotic system and avoids the need for a formal mandibulotomy. This reduces operation time and hospital stay with other potential benefits, including a more expedient return of swallow and potentially a reduced incidence of osteoradionecrosis at the osteotomy site. ${ }^{24}$

Selber ${ }^{25}$ described a small series of 5 patients undergoing oropharyngeal resection and reconstruction with an intact mandible with either local or free flaps. An anterolateral thigh flap was used in 1 patient to resurface the neck and reconstruct the tongue, floor of mouth and pharyngeal defects. The inset was performed using a combination of methods: by hand through the mouth and via a pharyngectomy and robotically via TORS for the more difficult to access areas. Microneedle drivers were employed to place the sutures but the investigators did comment that some of the knots ultimately were hand-tied. Several investigators since have described their techniques for salvage oropharyngectomies using both cervical and TORS approaches, with an emphasis on a combination of hand and robotic inset of free flaps (Paleri V, Fox $\mathrm{H}$, Coward S, et al. Transoral robotic surgery for residual and recurrent oropharyngeal cancers: an IDEAL phase 2a exploratory study of surgical innovation. In: Unpublished, ed., 2016). ${ }^{26-28}$

RAFFI is technically demanding and, in common with TORS ablation in the salvage setting, should be attempted only by experienced surgeons. Surgical time can be saved by performing resection and flap raising simultaneously but this requires a careful assessment of flap dimensions. This is best achieved using a combination of preoperative imaging and on-table measurements, with the need to accept that modifications may well be necessary as the case proceeds. To conform to the complex 3-dimensional ablative cavity, thin fasciocutaneous flaps are best employed. Time efficiency is maximized using a combination of a hand and robotic approaches for inset. In general, the flap is first supported by 2 or 3 holding sutures, 1 of which is 
placed cervically around the hyoid bone to stabilize the most inferior aspect of the flap and help achieve a watertight seal. The robotic inset should be performed first, because this allows any excess flap to be readily excised at its superior extent under direct vision. The lack of haptic feedback makes robotic tying of knots challenging and as such alternative sutures, such as the V-Loc barbed system (Medtronic, Watford, United Kingdom) have obvious advantages during inset, but the absorption profile of $\mathrm{V}$-Loc is a minimum of 90 days.

\section{Management of Positive Margins Recognized on Formalin-Fixed Paraffin-Embedded}

Difficult decisions need to be made patients in whom a paraffin section shows cancer in the defect margin after a negative frozen. For these patients, the margin should be considered to be positive and the authors believe that the surgeon has no option but to consider a re-resection. In many cases, it may be practically impossible to be precise about the site of the positive margin, especially in tongue base recurrences. In small tongue base ReRuNeR OPCs defects, a re-resection of the entire tumor bed may be feasible. In other instances, the sole option, the authors believe, is for open resection of the entire tumor bed and reconstruction as appropriate. In all such instances, in the authors' series ( 3 of the first 50 cases), no tumor was identified pathologically in the re-resected bed, but this could be a reflection of the minimal tumor volume and a sampling issue during processing.

\section{Postoperative Care}

The authors' policy for postoperative care is based on a patient comorbidity burden and requirement for postoperative ventilator support. Such patients are sent to the critical care units; all others return to the ward.

Pain is a significant component of the postoperative phase that needs to be actively managed to assist with the rehabilitation. The authors' postoperative TORS pain management protocol includes pregabalin, $150 \mathrm{mg}$ on the day of surgery, followed by $75 \mathrm{mg}$ twice a day, until the pain recedes and oral intake increases. Additionally, patient-controlled analgesia is used for 24 hours to 48 hours, with morphine given as required for breakthrough pain.

\section{Rehabilitation}

It has been well documented that swallowing function remains a primary concern for patients up to 12 months after definitive organ preservation treatment of head and neck cancer (HNC). ${ }^{29,30}$ Swallowing dysfunction has been shown to persist for many years after definitive treatment ${ }^{31}$ and for some patients can present as a late complication, with a gradual decline occurring many years after treatment. ${ }^{32}$ In the setting of ReRuNeR HNC, patients have the potential to present with a baseline dysphagia, related to their previous treatments, as well as new-onset dysphagia, as a symptom of their active disease. Dysphagia is associated with higher risk of pneumonia, poorer oral intake, prolonged gastrostomy use, poor nutritional status, weight loss, and significant alterations to eating patterns, social activities, and subsequently quality of life. ${ }^{33}$ Even in the setting of minimally invasive surgery for ReRuNeR OPCs, where more favorable functional outcomes have been reported in comparison to traditional surgical techniques (Paleri V, Fox H, Coward S, et al. Transoral robotic surgery for residual and recurrent oropharyngeal cancers: an IDEAL phase 2a exploratory study of surgical innovation. In: Unpublished, ed., 2016), ${ }^{34}$ a protracted period of dysphagia rehabilitation is required. ${ }^{35}$ Consequently, swallowing rehabilitation should be integral to the management of patients with ReRuNeR OPCs and should include 
prehabilitation with functional optimization before surgery. This should take place in addition to immediate postoperative therapy and followed by longer-term rehabilitation for the weeks and months after surgery. A rehabilitation model (Fig. 7) has been developed at the authors' center based on clinical experience and review of the existing literature in the management of dysphagia in HNC.

A multidimensional pretreatment swallowing evaluation underpins discussions regarding potential functional outcomes, as part of the informed consent process. $A$ thorough assessment also identifies targeted prehabilitation goals, including swallowing exercises. The baseline assessment should include a range of patient and clinician-reported measures, in addition to instrumental evaluation of swallowing function using videofluoroscopy and/or fiberoptic endoscopic evaluation of swallowing (FEES). ${ }^{35}$ Baseline evaluation also informs decision making regarding nonoral feeding methods in the acute and longer term after surgery. At the authors' center, an intraoperative nasogastric tube routinely is placed; however, depending on baseline swallowing function, some patients may require gastrostomy tube placement prior to surgery. If patients do not follow an expected trajectory of recovery (see Fig. 7), conversion from nasogastric tube to gastrostomy takes place at 14 days after surgery.

A range of rehabilitation approaches must be used, including targeted dysphagia swallowing exercises (see Fig. 7). More novel methods also have been used, including expiratory muscle strength training (EMST) and intensive blocks of boot camp-style interventions. ${ }^{35}$ The rehabilitation plan must be a patient-centered process, tailored to individual needs, with repeated outcome measures, allowing reactive changes to the plan as needed. ${ }^{36}$

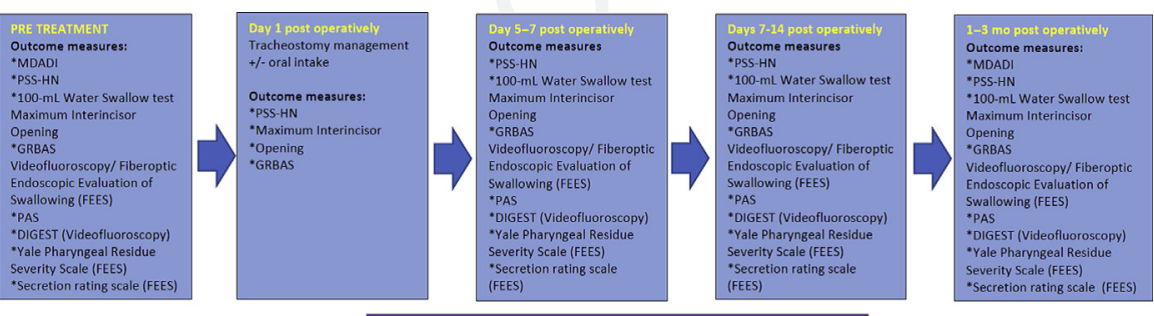

INTERVENTION
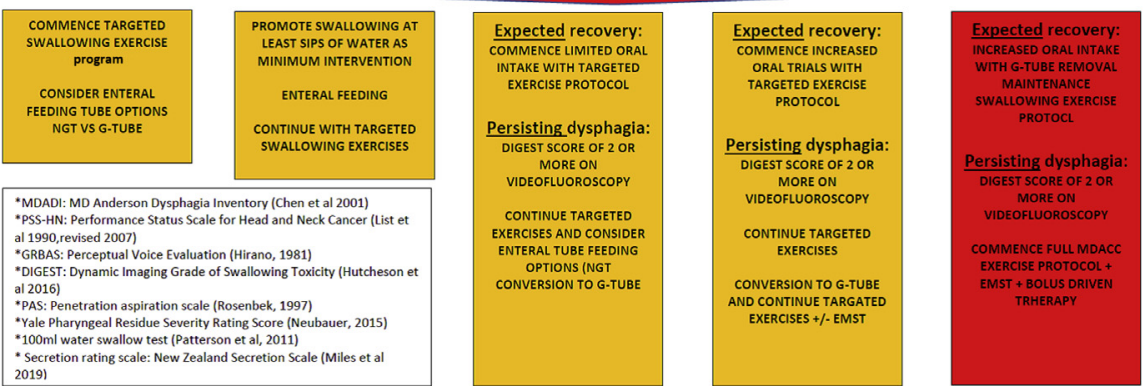

Fig. 7. Rehabilitation pathway for patients after salvage TORS. This rehabilitation model previously was presented at the Dysphagia Research Society. (From Brady GC, Leigh-Doyle, Q40 L, Stephen, S., Roe, J.W.G., Paleri, V. Functional Outcomes Following Transoral Robotic Surgery for Recurrent Head and Neck Cancer (HNC): A Prospectve Observational Study. In: Dysphagia, ed. Dysphagia Research Society 27th Anniversary Annual Meeting., 2019:9441018.) 


\section{Outcomes}

The authors' group performed a systematic review and meta-analysis of studies reporting survival data and functional outcomes for patients undergoing TORS for previously treated HNC. Of the 811 records identified, 8 were eligible for inclusion, covering 165 cases (range 1-64). There was a male preponderance, and the mean ages were approximately 60 years. Nearly all cases were squamous cell carcinoma, but HPV rates were reported inconsistently. Most cases were early-stage disease, Q25 rTO-T2 and rNO-N2b.

The pooled free flap rate was $0.9 \%$ (4 studies; range $0.0-14.3 ; 95 \% \mathrm{Cl}, 0.0-6.8 ; \mathrm{I}^{2}$ $63.7 \% ; P=.04)$.

\section{COMPLICATIONS}

The meta-analysis showed a pooled postoperative hemorrhage rate of $9.2 \%$ from 4 studies (range 3.3-13.3), with a pooled postoperative pharyngocutaneous fistula rate of $0.6 \%$ (4 studies, range $0.0-3.3$ ).

\section{MARGINS}

All but 1 study reported on rates of positive resection margins, with 5 studies also reporting rates of close resection margins. The pooled positive margin rate was $18.2 \%$ (4 studies, range 6.7-33.3). The pooled close margin rate (not including positive margins) was $25.7 \%$ (3 studies, range 6.7-52.9). The criteria used for a close margin cutoff was reported by 4 studies, ranging between $2 \mathrm{~mm}$ and $5 \mathrm{~mm}$, with only 1 study reporting the criteria used for considering a margin as positive.

\section{ONCOLOGIC OUTCOMES}

The pooled data for oncological outcomes (Fig. 8) were as follows: 2-year overall survival, 73.1\% (4 studies; range 64.7-75.0; 95\% Cl, 64.6-80.9; $\mathrm{I}^{2} 0.0 \% ; P=.9$ ), and 2year disease-free survival, 75.3\% (4 studies; range 60.0-92.0; 95\% Cl, 65.2-84.2; $\mathrm{I}^{2}$ $22.9 \% ; P=.3)$.

\section{FUNCTIONAL OUTCOMES}

Only surrogate functional outcomes were available from the systematic review. The pooled perioperative gastrostomy rate from 3 studies was 25.0\% (range 16.7-35.9), with a pooled perioperative tracheostomy rate of $22.3 \%$ (3 studies; range 21.923.5). Some long-term results were available for functional outcomes, although the definitions of what constituted long-term outcomes were not clear in the source material. The pooled long-term gastrostomy rate was 5.0\% (4 studies; range $0.0-20.0$ ) and the pooled long-term tracheostomy rate was $1.9 \%$ (2 studies; range $0.0-10.0$ ), indicating that a vast majority of patients were swallowing without tubes and were decannulated in the longer term.

To provide more granular data, the authors present their center results for functional outcomes. Between December 2017 and August 2019, 30 patients (4 women) underwent TORS for ReRuNeR. Previous treatments included (biochemo)radiation $(n=28)$ and surgery with postoperative radiotherapy $(n=2)$. Median age was 60 years (range 37-82 years). Patients had locally recurrent/residual disease of the oropharynx $(n=29)$ and hypopharynx $(n=2)$. TORS-assisted flap reconstruction was required in 8 patients. Tracheostomy was performed in 25 patients. Median time to decannulation was 11 days (range 4-27). Baseline and postsurgery gastrostomy use was as 


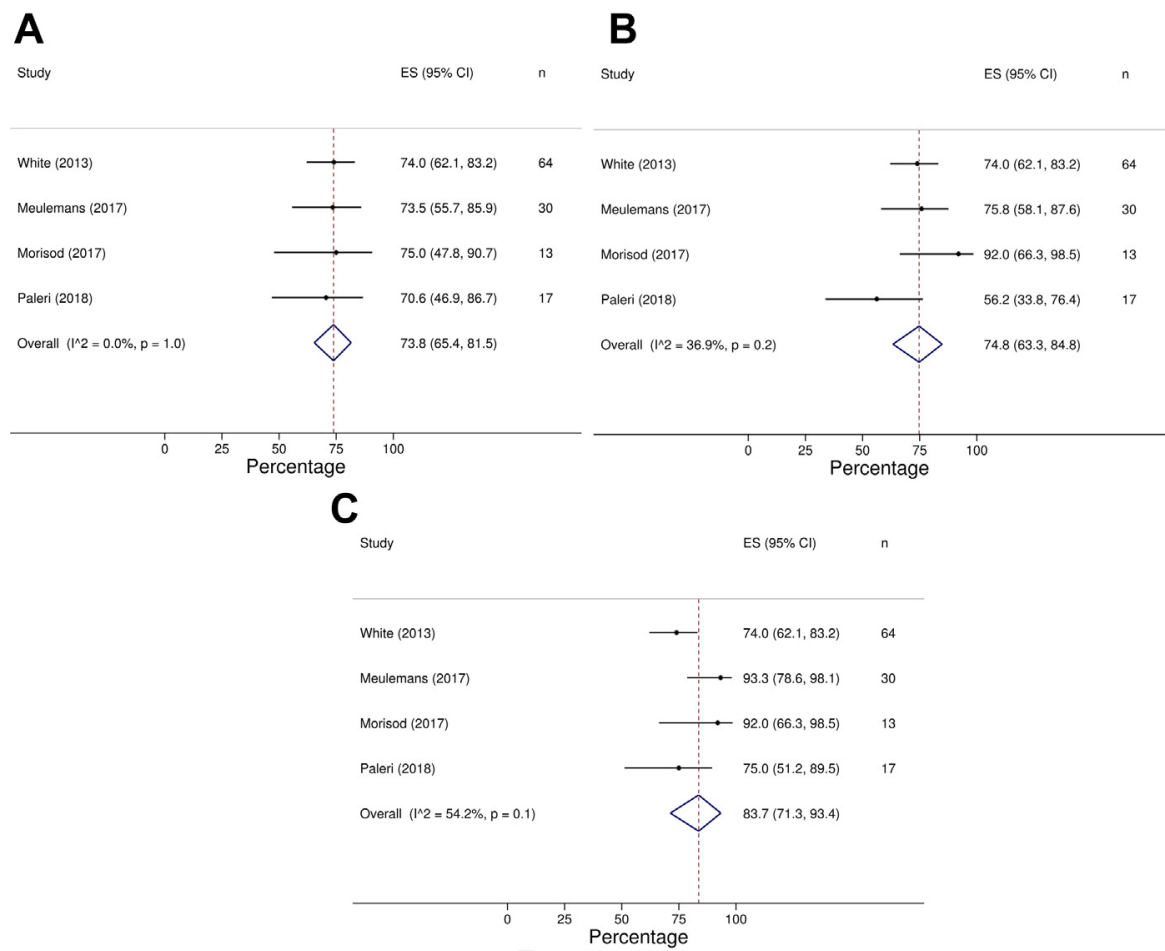

Fig. 8. Pooled 2-year overall survival $(A)$, disease-free survival $(B)$, and disease-specific survival (C) after salvage TORS for ReRuNeR cancers. (From Hardman J, Liu Z, Brady Get al. Transoral robotic surgery for recurrent cancers of the upper aerodigestive tract-Systematic review and meta-analysis. Head Neck 2020; 42:1089-1104.)

follows: 6 at baseline $(n=30) ; 15$ at 3 months $(n=30) ; 7$ at 6 months ( $n=16$ assessable patients); and 4 at 12 months postsurgery ( $n=10$ assessable patients). Median length of hospital stay was 14 days (range 1-30). Further objective and patientreported outcome measures, including the MD Anderson Dysphagia Inventory (MDADI) (Chen and colleagues, 2001), Performance Status Scale for Head and ${ }_{226}$ Neck Cancer Patients Normalcy of Diet (PSS-HN) (List and colleagues, 1990), and a27 maximum interincisor opening, are shown in Table 1.

\section{FUTURE TRENDS}

Revising margins after salvage TORS is a difficult prospect, so real-time assessment of margins and revision of the resection at the time of surgery are key to avoiding this predicament. Frozen sections are associated with an inevitable delay between sampling and the subsequent result, which may result in difficulty relating any positive results to precise location in the resection bed, given the complexity of the endoscopic landscape. Real-time assessment of the margins might be the way forward in this setting and several options have shown promise in this regard. Rapid evaporation ionizing mass spectrometry, where analysis of the plume from the area being cut by the electrocautery instrument, has shown promise and is one option that has been robustly validated in the laboratory setting. ${ }^{37}$ The authors' work on snap frozen samples from 74 patients and 1051 observations offers the following diagnostic efficacy 
712

713

714

715

716

717

718

719

720

721

722

723

724

725

726

727

728

729

730

731

732

733

734

735

736

737

738

739

740

741

742

743

744

745

746

747

748

749

750

751

752

753

754

755

756

757

758

759

760

761

762

\begin{tabular}{|c|c|c|c|c|}
\hline \multicolumn{5}{|c|}{$\begin{array}{l}\text { Table } 1 \\
\text { Functional outcome measures from a single center cohort }\end{array}$} \\
\hline & Baseline & $\begin{array}{l}\text { Three } \\
\text { Months Post }\end{array}$ & $\begin{array}{l}\text { Six Months } \\
\text { Post }\end{array}$ & $\begin{array}{l}\text { Twelve Months } \\
\text { Post }\end{array}$ \\
\hline Mean PSS-HN & 69.3 & 39.3 & 51.2 & 51.1 \\
\hline $\begin{array}{l}\text { Normalcy of } \\
\text { diet score }\end{array}$ & $\begin{array}{l}95 \% \mathrm{Cl}_{1} \\
62.0-76.7 \\
\mathrm{n}=30\end{array}$ & $\begin{array}{l}95 \% \mathrm{Cl} \\
29.9-48.7 \\
\mathrm{n}=29\end{array}$ & $\begin{array}{l}95 \% \mathrm{Cl}_{1} \\
37.6-68.4 \\
\mathrm{n}=17\end{array}$ & $\begin{array}{l}95 \% \mathrm{Cl} \\
30.1-72.1 \\
\mathrm{n}=9\end{array}$ \\
\hline $\begin{array}{l}\text { Mean MDADI } \\
\text { composite score }\end{array}$ & $\begin{array}{l}74.6 \\
95 \% \mathrm{Cl} \\
68-81.2 \\
\mathrm{n}=24 \\
\end{array}$ & $\begin{array}{l}45.6 \\
95 \% \mathrm{Cl} \\
\quad 32.7-58.5 \\
\mathrm{n}=12\end{array}$ & $\begin{array}{l}59.5 \\
95 \% \mathrm{Cl} \\
46.3-72.7 \\
\mathrm{n}=11 \\
\end{array}$ & $\begin{array}{l}61.8 \\
95 \% \mathrm{Cl}, \\
34.7-88.9 \\
\mathrm{n}=6 \\
\end{array}$ \\
\hline $\begin{array}{l}\text { Median maximum } \\
\text { interincisor opening }(\mathrm{mm})\end{array}$ & $\begin{array}{l}38.5 \\
\text { Range: } 20-55 \\
n=26\end{array}$ & $\begin{array}{l}26.5 \\
\text { Range: } 10-43 \\
n=22\end{array}$ & $\begin{array}{l}31 \\
\text { Range: } 10-50 \\
n=9\end{array}$ & \\
\hline
\end{tabular}

metrics: specificity, 98.47\%; sensitivity, 97.78\%; positive predictive value, $97.4 \%$; and negative predictive value, $98.4 \%$. This innovative technology will greatly increase intraoperative confidence in the adequacy of the resection margins and is under ongoing evaluation.

Although immunotherapy approaches have shown success compared with conventional chemotherapy for unresectable nonmetastatic cancers, ${ }^{38}$ surgery remains the sole curative option. No targeted nonsurgical therapies are available on the horizon. A recent prospective trial to assess the clinical benefit of a tailored gene set built on a next-generation sequencing platform in patients with recurrent or metastatic head and neck squamous cell carcinoma provided to clinicians to inform treatment decisions did not provide clinical benefit to the patients. ${ }^{39}$ Promising avenues of investigation appear to be combining immunotherapy in the surgically salvageable population too (ClinicalTrials.gov Identifier: NCT03565783).

\section{DISCUSSION}

TORS clearly is proving itself an acceptable treatment modality for recurrent cancers, with outcomes comparable to the results of open resections ${ }^{2}$ and transoral laser resections. ${ }^{40,41}$ The caveats associated with these outcomes must be interpreted carefully, however; there is a selection bias to those being offered TORS. This bias pertains mainly to 2 factors: (1) the selection of relatively smaller cancers (approximately 90\%) is limited to 1 subsite vs larger tumors, and (2) smaller recurrences usually are HPVpositive cancers in patients with a better comorbidity profile compared with to HPVnegative squamous cell cancers. ${ }^{42}$ This cohort usually has a greater respiratory reserve and tolerates larger transoral resections, which still leave them with a functional swallow. The authors hope to have greater clarity on these aspects in a forthcoming international individual patient data meta-analysis that currently is under way (IRAS 268830, RMHCCR5156).

TORS should form just 1 modality in the spectrum of treatments available to patients with ReRuNeR OPCs. Although transoral laser microsurgery (TLM) for ReRuNeR Q29 OPCs is not to be discounted entirely, it is the authors' experience that TLM is unsuited for anything but the smallest tonsil cancers. The ability to perform intraoperative imaging and flap inset are unique to TORS resections, expanding the patient base for transoral surgery. 
As a surgeon's practice and experience evolve, it is likely that the patients deemed suitable for these procedures will expand too. The authors emphasize that prior to embarking on any TORS for ReRuNeR cancer, the members of their multidisciplinary team carefully consider all aspects of a patient's treatment, from surgical to oncological to functional perspectives, to ensure the patient may be counseled appropriately. The postoperative complication profile and the long-term rehabilitation outcomes are significantly different from those of the primary cohort and need to be understood when embarking on this service. The authors' hospital stay data may be skewed by patients from outside the region; discharge policy allows patients to return directly home (rather than to an interim closer hospital) only after the following criteria are met: the tracheostomy is decannulated; pain is well controlled; nutritional requirements are met through oral intake and/or an appropriate feeding tube; nursing support is in place for the feeding tube and any social care issues; and patient transport can be arranged to the referring area of the country. The authors' previous single-center data indicate a hospital stay of under a week when patients receive treatment at their local center and many of these logistical issues do not apply (Paleri V, Fox H, Coward S, et al. Transoral robotic surgery for residual and recurrent oropharyngeal cancers: an IDEAL phase 2a exploratory study of surgical innovation. In: Unpublished, ed., 2016).

\section{DISCLOSURE}

V. Paleri is a proctor for Intuitive Surgical Si and Xi systems.

\section{REFERENCES}

1. Culie D, Benezery K, Chamorey E, et al. Salvage surgery for recurrent oropharyngeal cancer: post-operative oncologic and functional outcomes. Acta Otolaryngol 2015;135:1323-9.

2. Jayaram SC, Muzaffar SJ, Ahmed I, et al. Efficacy, outcomes, and complication rates of different surgical and nonsurgical treatment modalities for recurrent/residual oropharyngeal carcinoma: A systematic review and meta-analysis. Head Neck 2016;38:1855-61.

3. Nichols AC, Kneuertz PJ, Deschler DG, et al. Surgical salvage of the oropharynx after failure of organ-sparing therapy. Head Neck 2011;33:516-24.

4. Patel SN, Cohen MA, Givi B, et al. Salvage surgery for locally recurrent oropharyngeal cancer. Head Neck 2016;38(Suppl 1):E658-64.

5. Hay A, Simo R, Hall G, et al. Outcomes of salvage surgery for the oropharynx and larynx: a contemporary experience in a UK Cancer Centre. Eur Arch Otorhinolaryngol 2019;276:1153-9.

6. Maruo T, Zenda S, Shinozaki T, et al. Comparison of salvage surgery for recurrent or residual head and neck squamous cell carcinoma. Jpn J Clin Oncol 2020;50: 288-95.

7. Fakhry C, Zhang Q, Nguyen-Tan PF, et al. Human papillomavirus and overall survival after progression of oropharyngeal squamous cell carcinoma. J Clin Oncol 2014;32:3365-73.

8. Daly ME, Le QT, Maxim PG, et al. Intensity-modulated radiotherapy in the treatment of oropharyngeal cancer: clinical outcomes and patterns of failure. Int J Radiat Oncol Biol Phys 2010;76:1339-46.

9. Garden AS, Dong L, Morrison WH, et al. Patterns of disease recurrence following treatment of oropharyngeal cancer with intensity modulated radiation therapy. Int J Radiat Oncol Biol Phys 2013;85:941-7. 
814

815

816

817

818

819

820

821

822

823

824

825

826

827

828

829

830

831

832

833

834

835

836

837

838

839

840

841

842

843

844

845

846

847

848

849

850

851

852

853

854

855

856

857

858

859

860

861

862

863

864
10. Goldenberg D, Begum S, Westra WH, et al. Cystic lymph node metastasis in patients with head and neck cancer: An HPV-associated phenomenon. Head Neck 2008;30:898-903.

11. Hardman J, Liu Z, Brady G, et al. Transoral robotic surgery for recurrent cancers of the upper aerodigestive tract-Systematic review and meta-analysis. Head Neck 2020;42:1089-104.

12. Albergotti WG, Gooding WE, Kubik MW, et al. Assessment of Surgical Learning Curves in Transoral Robotic Surgery for Squamous Cell Carcinoma of the Oropharynx. JAMA Otolaryngol Head Neck Surg 2017;143:542-8.

13. Weinstein GS, O'Malley BW Jr. Transoral robotic surgery (TORS). San Diego (CA): Plural Publishing Inc.; 2011.

14. Mohamed A, Paleri V, George A. A cadaveric study quantifying the anatomical landmarks of the facial artery and its parapharyngeal branches for safe transoral surgery. Head Neck 2019;41:3389-94.

15. Wang C, Kundaria S, Fernandez-Miranda J, et al. A description of arterial variants in the transoral approach to the parapharyngeal space. Clin Anat 2014;27: 1016-22.

16. Laccourreye O, Orosco RK, Rubin F, et al. Styloglossus muscle: a critical landmark in head and neck oncology. Eur Ann Otorhinolaryngol Head Neck Dis 2018;135:421-5.

17. Weinstein GS, O'Malley BW Jr, Snyder W, et al. Transoral robotic surgery: radical tonsillectomy. Arch Otolaryngol Head Neck Surg 2007;133:1220-6.

18. Clayburgh DR, Byrd JK, Bonfili J, et al. Intraoperative Ultrasonography During Transoral Robotic Surgery. Ann Otol Rhinol Laryngol 2016;125:37-42.

19. Johnson RE, Sigman JD, Funk GF, et al. Quantification of surgical margin shrinkage in the oral cavity. Head Neck 1997;19:281-6.

20. Mistry RC, Qureshi SS, Kumaran C. Post-resection mucosal margin shrinkage in oral cancer: quantification and significance. J Surg Oncol 2005;91:131-3.

21. Song HG, Yun IS, Lee WJ, et al. Robot-assisted free flap in head and neck reconstruction. Arch Plast Surg 2013;40:353-8.

22. de Almeida JR, Park RC, Villanueva NL, et al. Reconstructive algorithm and classification system for transoral oropharyngeal defects. Head Neck 2014;36: 934-41.

23. de Almeida JR, Park RC, Genden EM. Reconstruction of transoral robotic surgery defects: principles and techniques. J Reconstr Microsurg 2012;28:465-72.

24. Lee SY, Park YM, Byeon HK, et al. Comparison of oncologic and functional outcomes after transoral robotic lateral oropharyngectomy versus conventional surgery for T1 to T3 tonsillar cancer. Head Neck 2014;36:1138-45.

25. Selber JC. Transoral robotic reconstruction of oropharyngeal defects: a case series. Plast Reconstr Surg 2010;126:1978-87.

26. Chan JYW, Chan RCL, Chow VLY, et al. Transoral robotic total laryngopharyngectomy and free jejunal flap reconstruction for hypopharyngeal cancer. Oral Oncol 2017;72:194-6.

27. Gorphe P, Temam S, Kolb F, et al. Cervical-transoral robotic oropharyngectomy and thin anterolateral thigh free flap. Eur Ann Otorhinolaryngol Head Neck Dis 2018;135:71-4.

28. Mukhija VK, Sung CK, Desai SC, et al. Transoral robotic assisted free flap reconstruction. Otolaryngol Head Neck Surg 2009;140:124-5.

29. Wilson JA, Carding PN, Patterson JM. Dysphagia after nonsurgical head and neck cancer treatment: patients' perspectives. Otolaryngol Head Neck Surg $2011 ; 145: 767-71$. 
30. Roe JW, Drinnan MJ, Carding PN, et al. Patient-reported outcomes following parotid-sparing intensity-modulated radiotherapy for head and neck cancer. How important is dysphagia? Oral Oncol 2014;50:1182-7.

31. Patterson JM. Late Effects of Organ Preservation Treatment on Swallowing and Voice; Presentation, Assessment, and Screening. Front Oncol 2019;9:401.

32. Cohen EE, LaMonte SJ, Erb NL, et al. American Cancer Society Head and Neck Cancer Survivorship Care Guideline. CA Cancer J Clin 2016;66:203-39.

33. Patterson JM, Brady GC, Roe JW. Research into the prevention and rehabilitation of dysphagia in head and neck cancer: a UK perspective. Curr Opin Otolaryngol Head Neck Surg 2016;24:208-14.

34. White H, Ford S, Bush B, et al. Salvage surgery for recurrent cancers of the oropharynx: comparing TORS with standard open surgical approaches. JAMA Otolaryngol Head Neck Surg 2013;139:773-8.

35. Brady GC, Hardman JC, Paleri V, et al. Changing paradigms in the treatment of residual/recurrent head and neck cancer: implications for dysphagia management. Curr Opin Otolaryngol Head Neck Surg 2020;28(3):165-71.

36. Wade DT. What is rehabilitation? An empirical investigation leading to an evidence-based description. Clin Rehabil 2020;34(5):571-83.

37. Dhanda J, Schache A, Robinson M, et al. iKnife Rapid Evaporative Ionisation Mass Spectrometry (REIMS) Technology In Head and Neck Surgery. A Ex Vivo Feasibility Study. Br J Oral Maxillofac Surg 2017.

38. Ferris RL, Blumenschein $\mathrm{G} J \mathrm{~J}$, Fayette $\mathrm{J}$, et al. Nivolumab for Recurrent Squamous-Cell Carcinoma of the Head and Neck. N Engl J Med 2016;375: 1856-67.

39. Westbrook TC, Hagemann IS, Ley J, et al. Prospective assessment of the clinical benefit of a tailored cancer gene set built on a next-generation sequencing platform in patients with recurrent or metastatic head and neck cancer. Med Oncol 2019;37:12.

40. Grant DG, Salassa JR, Hinni ML, et al. Carcinoma of the tongue base treated by transoral laser microsurgery, part two: Persistent, recurrent and second primary tumors. Laryngoscope 2006;116:2156-61.

41. Melong JC, Rigby MH, Bullock M, et al. Transoral laser microsurgery for the treatment of oropharyngeal cancer: the Dalhousie University experience. J Otolaryngol Head Neck Surg 2015;44:39.

42. Hess CB, Rash DL, Daly ME, et al. Competing causes of death and medical comorbidities among patients with human papillomavirus-positive vs human papillomavirus-negative oropharyngeal carcinoma and impact on adherence to radiotherapy. JAMA Otolaryngol Head Neck Surg 2014;140:312-6. 


\section{AUTHOR QUERY FORM}

\begin{tabular}{|c|c|}
\hline 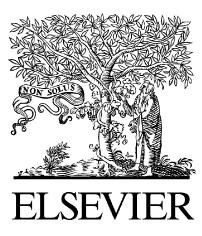 & $\begin{array}{l}\text { Journal: OTC } \\
\text { Article Number: } 1813\end{array}$ \\
\hline
\end{tabular}

Dear Author,

Please check your proof carefully and mark all corrections at the appropriate place in the proof (e.g., by using on-screen annotation in the PDF file) or compile them in a separate list. It is crucial that you NOT make direct edits to the PDF using the editing tools as doing so could lead us to overlook your desired changes. Note: if you opt to annotate the file with software other than Adobe Reader then please also highlight the appropriate place in the PDF file. To ensure fast publication of your paper please return your corrections within 48 hours.

For correction or revision of any artwork, please consult http://www.elsevier.com/artworkinstructions.

Any queries or remarks that have arisen during the processing of your manuscript are listed below and highlighted by flags in the proof.

\begin{tabular}{|c|c|}
\hline $\begin{array}{l}\text { Location } \\
\text { in article }\end{array}$ & $\begin{array}{l}\text { Query / Remark: Click on the } Q \text { link to find the query's location in text } \\
\text { Please insert your reply or correction at the corresponding line in the proof }\end{array}$ \\
\hline Q1 & $\begin{array}{l}\text { Please verify your preferred correspondence address to be published and provide any missing } \\
\text { information. Elsevier recommends not using your personal home address. }\end{array}$ \\
\hline Q2 & $\begin{array}{l}\text { For your co-authors, please verify their affiliations and provide a complete address for the affiliations } \\
\text { listed. The address will appear on the footnote of the first page of your article and will be published. Once } \\
\text { again, Elsevier recommends not using personal home addresses. Also, please note that we will send each } \\
\text { contributing author a copy of this issue to their mentioned address. }\end{array}$ \\
\hline Q3 & Please approve the short title to be used in the running head at the top of each right-hand page. \\
\hline Q4 & As per the editorial remarks, "Please add a list of Clinics Care Points to the manuscript." \\
\hline Q5 & $\begin{array}{l}\text { Degree abbreviations are verified against a list of known degrees. MRes, MRCSLT are not yet on this list. } \\
\text { Please verify these degrees. }\end{array}$ \\
\hline Q6 & Are author names and order of authors OK as set? \\
\hline Q7 & $\begin{array}{l}\text { This is how your names will appear on the contributors' list. Please add your academic titles, if missing, } \\
\text { as well as any other necessary titles and professional affiliations. } \\
\text { VINIDH PALERI, MS, FRCS, Consultant Head and Neck Surgeon, Head and Neck Unit, The Royal } \\
\text { Marsden NHS Foundation Trust, Professor of Robotic and Endoscopic Head and Neck Surgery, The } \\
\text { Institute of Cancer Research, London, United Kingdom } \\
\text { JOHN HARDMAN, MSc, MRCS, Head and Neck Unit, The Royal Marsden NHS Foundation Trust, } \\
\text { London, United Kingdom; Specialty Registrar in ENT, North London, United Kingdom } \\
\text { GRAINNE BRADY, MRes, MRCSLT, Clinical Lead Speech \& Language Therapist, Department of } \\
\text { Speech, Language and Swallowing, The Royal Marsden NHS Foundation Trust, London, United Kingdom } \\
\text { AJITH GEORGE, FRCS, Consultant Head and Neck Surgeon, University Hospitals North Midlands, } \\
\text { North Staffordshire, England; Senior Lecturer, Keele University Medical School, Staffordshire, United }\end{array}$ \\
\hline
\end{tabular}


CYRUS KERAWALA, FDSRCS, FRCS, Consultant Head and Neck Surgeon, Head and Neck Unit, The Royal Marsden NHS Foundation Trust, London, United Kingdom

The following synopsis is the one that you supplied but lightly copyedited. Please confirm OK. Please note that the synopsis will appear in PubMed: Transoral robotic surgery (TORS) is a well-established treatment option for treatment-naïve oropharyngeal cancer. For residual, recurrent, and new primary oropharyngeal tumors emerging in previously irradiated fields, the global experience of management with TORS is limited. This article discusses current concepts on this topic, offers a deeper insight into the transoral anatomy for these cases, and covers the specific complexities of resections in the various subsites of the oropharynx. It provides practical tips on reconstruction, recovery, and rehabilitation as well as offering a synthesis of the current evidence and exploring future trends.

Please verify edit, "may be required".

Q10

Q11

Q12

Q13

Q14

Q15

Q16

Q17

Q18

Q19

Q20

Q21

Q22

Q23

Q24

Q25

Q26

Q27

Q28

Q29

Q30

Q31

Please check the hierarchy of the section headings.

If there are any drug dosages in your article, please verify them and indicate that you have done so by initialing this query.

Please verify IMRT expansion.

Per style, personal pronouns avoided; please verify edits, "authors", throughout.

Please verify/clarify that "principles of" refers only to "case selection" and "reconstruction".

Can "T" be "T-stage"?

Please verify edits, "The subdivisions...".

Please verify that 14 headings, "Neck and Airway" through "Outcomes" are all subheadings below "Surgical technique and refinements".

Please verify edit, "FK-WO", throughout. Add manufacturers and locations for trade names?

Please verify sue of “inch" (in) vs usual Clinics SI style, $\mathrm{cm}$ or $\mathrm{mm}$.

Sections are not numbered; please modify "section 5.4" as either "discussed later" or "discussed previously".

Please verify FFPE expansion; and, discuss in text? And, add a term, eg "Tissue" or "Specimen"?

Please verify if "frozen" is complete term.

Please specify "Such patients".

Should "patient" be "patient-reported"?

Please verify SCC expansion.

Please modify "Chen and colleagues, 2001" and "List and colleagues, 1990" as ref. numbers.

Please verify that "Normalcy of Diet" is part of "PSS-HN".

Please clarify what "too" refers to.

Please verify TLM expansion.

Please verify edit, "different from those of"; and, verify that clear what "primary cohort" refers to.

Correctly acknowledging the primary funders and grant IDs of your research is important to ensure compliance with funder policies. We could not find any acknowledgement of funding sources in your text. Is this correct? 


\begin{tabular}{|l|l|} 
Q32 & Table 1, please verify if "Post" is complete term in column headings. \\
Q33 & Please verify the layout of Table 1, and correct if necessary. \\
Q35 & Please provide the volume number or issue number and page range in Ref. 37. \\
Q36 & Fig. 2 legend, please add expansions for APa, Fa, Inf, Lat, Med, SGM, SPM, Sup, and TA. \\
Q37 & $\begin{array}{l}\text { Fig. } 4 \text { legend, please verify adding “(CN)". Please add expansion for a, m, BULB, SGM, Sup. } \\
\text { Q38 }\end{array}$ \\
Q39. 5 legend, please verify edit, "have been". \\
Q40 & $\begin{array}{l}\text { Fig. 6 legend, please add expansions for A, I, L, M. } \\
\text { FGS. }\end{array}$ \\
& $\begin{array}{l}\text { Please check this box or indicate } \\
\text { your approval if you have no } \\
\text { corrections to make to the PDF file }\end{array}$ \\
&
\end{tabular}

Thank you for your assistance. 